\title{
Texturing degree boosts thermoelectric performance of silver-doped polycrystalline SnSe
}

\author{
Xue Wang ${ }^{1,2}$, Jingtao $\mathrm{Xu}^{1}$, Guo-Qiang Liu ${ }^{1}$, Xiaojian $\mathrm{Tan}^{1}$, Debo $\mathrm{Li}^{1}$, Hezhu Shao ${ }^{1}$, Tianya Tan ${ }^{2}$ \\ and Jun Jiang ${ }^{1}$
}

Tin selenite ( $\mathrm{SnSe}$ ) has attracted significant attention due to its record thermoelectric figure of merit ( $Z T=2.6$ at $923 \mathrm{~K}$ ) of its single crystal. However, the polycrystalline SnSe processes considerably less ZTs $(\leqslant 1.1)$. In this study, we investigate the thermoelectric properties of Ag-doped polycrystalline SnSe, which was synthesized via zone melting and hot pressing. By comparing our results and previous reports of Ag-doped single crystals and polycrystals, we determine that the high texturing degree is essential for achieving good thermoelectric performance in polycrystalline $\mathrm{SnSe}$. The zone-melted $\mathrm{Sn}_{0.99} \mathrm{Ag}_{0.02} \mathrm{Se}$ shows better thermoelectric performance than the Ag-doped SnSe single crystal in the entire temperature range, exhibiting a peak $Z T$ of 1.3 at $793 \mathrm{~K}$.

NPG Asia Materials (2017) 9, e426; doi:10.1038/am.2017.147; published online 25 August 2017

\section{INTRODUCTION}

Thermoelectric materials, which are able to directly convert heat energy into electrical power, have received global attention. ${ }^{1-4}$ The efficiency of thermoelectric devices is determined via the dimensionless figure of merit, which is defined as $Z T=S^{2} \sigma T / \kappa$, where $S$ is the Seebeck coefficient, $\sigma$ is the electrical conductivity, $\kappa$ is the thermal conductivity and $T$ is the absolute temperature. The thermoelectric performance has been significantly improved in the past two decades through new understanding of transport properties, ${ }^{5-8}$ and $Z T$ records have been continuously renewed in thermoelectric materials. ${ }^{6-10}$

Recently, the IV-VI compound SnSe has received considerable attention because of its ultrahigh thermoelectric performance in both undoped and p-type-doped single crystals (SCs). ${ }^{11-13}$ SnSe has a layered structure (with a Pnma space group, \#62 at room temperature) and strong anisotropic properties. The $a$ axis possesses an ultralow thermal conductivity but low ZTs. High thermoelectric performance is observed in the $b c$ plane, $Z T=2.62$ at $923 \mathrm{~K}$ along the $b$ axis and 2.3 along the $c$ axis. The high thermoelectric performance of SnSe is attributed to its low thermal conductivity, as low as $0.23 \mathrm{~W} \mathrm{~m}^{-1} \mathrm{~K}$ at $973 \mathrm{~K} .{ }^{11}$ Recently, several authors have argued that the reported ultralow thermal conductivity is due to the poor density of samples, and it is not intrinsic. ${ }^{14}$ Because of poor machinability and difficulties of growing SCs, increasing attention has been paid to its polycrystalline counterparts.

Polycrystalline samples exhibit a considerably worse thermoelectric performance than the SC. The highest reported $Z T$ for polycrystalline SnSe is only 1.1, which is less than half of the undoped SC. ${ }^{15,16}$ Even though alloying and microstructure modulation have been used to reduce thermal conductivity, polycrystalline samples exhibit a higher $\kappa$ than the SC, ${ }^{15-20}$ which contradicts phonon scattering considering the effect of grain boundary scattering. In addition, most polycrystalline samples possess lower power factors $\left(S^{2} \sigma\right)$ than the SC, which suggests that the control of crystallite orientation is very important in polycrystalline SnSe samples. ${ }^{15-20}$

The undoped SnSe shows a carrier concentration of $\sim 10^{17} \mathrm{~cm}^{-3}, 11$ which is considerably lower than the optimal region for a thermoelectric material. ${ }^{2-4}$ Therefore, chemical doping has been used to tune the carrier concentration and improve the thermoelectric performance of SnSe. Ag, ${ }^{21,22} \mathrm{Na}^{23-25}$ and $\mathrm{K}^{26}$ have been used to increase hole concentration, and $\mathrm{I}^{27}$ and $\mathrm{BiCl}_{3}{ }^{28}$ have been used as n-type dopants. Significant enhancement in $Z T$ values below the phase transition was observed in doped SCs. ${ }^{12,13}$ However, the doped polycrystalline samples still show $Z T$ values that are less than 1.1, which is the highest reported value of undoped polycrystalline samples, although the carrier concentration has been optimized to $>10^{19} \mathrm{~cm}^{-3} \cdot{ }^{19-30}$ The deep understanding of electrical and thermal transport properties and exquisite control of the crystallite orientation in the samples are essential to achieve high thermoelectric performance in polycrystalline SnSe.

In this study, we prepare an Ag-doped polycrystalline SnSe via hot pressing (HP) and zone melting ( $\mathrm{ZM})$. We compare the thermoelectric properties of our samples with an Ag-doped SC and polycrystalline samples via spark plasma sintering (SPS). ${ }^{13,22} \mathrm{We}$ found that the sample prepared via ZM show excellent electrical properties and lower thermal conductivity compared with the SC. Our

${ }^{1}$ Ningbo Institute of Materials Technology and Engineering, Chinese Academy of Sciences, Ningbo 315201, China and ${ }^{2}$ Department of Physics, Liaoning University, Shenyang 110036, China

Correspondence: Professor J Xu or Professor J Jiang, Ningbo Institute of Materials Technology and Engineering, Chinese Academy of Sciences, 1219 West Zhongguan Road, Zhenhai, Ningbo 315201, China.

E-mail: xujingtao@nimte.ac.cn or jjun@nimte.ac.cn

Received 26 December 2016; revised 14 May 2017; accepted 18 June 2017 
results suggest that better thermoelectric performance can be achieved in a highly textured polycrystalline SnSe.

\section{MATERIALS AND METHODS}

High-purity starting materials, Sn granules (99.999\%), Se granules (99.999\%) and Ag granules (99.9\%), were weighed according to the stoichiometry of $\mathrm{Sn}_{1-x} \mathrm{Ag}_{2 x} \mathrm{Se}(x=0,0.01,0.02)$ and sealed under high vacuum $\left(\sim 10^{-3} \mathrm{~Pa}\right)$ in quartz tubes. The mixtures were melted at $1223 \mathrm{~K}$ for $1 \mathrm{~h}$ in a rocking furnace to ensure homogeneity of the composition. Next, the mixtures were airquenched. For HP, the obtained cylindrical ingots were crushed by hand and ground into fine powders with an agate pestle and mortar. The powders were loaded into a graphite mold with a $12.7-\mathrm{mm}$ diameter and sintered under a pressure of $60 \mathrm{MPa}$ for $30 \mathrm{~min}$ at $753 \mathrm{~K}$. For $\mathrm{ZM}$, the quartz tubes after rocking were put into a $\mathrm{ZM}$ furnace, and the crystals were grown at $1223 \mathrm{~K}$ with a constant speed of $10.66 \mathrm{~mm} \mathrm{~h}^{-1} .15$

$\mathrm{X}$-ray diffraction (XRD) analysis was performed in a reflection geometry using a diffractometer (D8 Advance, Bruker, Kalsruhe, Germany). The analysis was performed on the samples that were parallel/perpendicular to the applied pressure or growth direction. The microstructures were investigated using a scanning electron microscope (SEM; Quanta FEG 250, FEI, Hillsboro, OR, USA) with an attached energy dispersive spectrometer (Inca X-Max, Oxford Instruments, Oxford, England). The actual composition of the samples was determined using the electron probe micro-analysis (EPMA-1610, Shimadzu, Kyoto, Japan).

The thermoelectric properties were measured along the (400)-preferred orientation. According to previous studies, ${ }^{15}$ the hot pressed samples were cut perpendicular to the pressing direction, and the zone-melt sample was cut along the growing direction. For electrical measurements, the samples were cut into $8 \mathrm{~mm} \times 2 \mathrm{~mm} \times 2 \mathrm{~mm}$ bars. The Seebeck coefficient and electrical con-

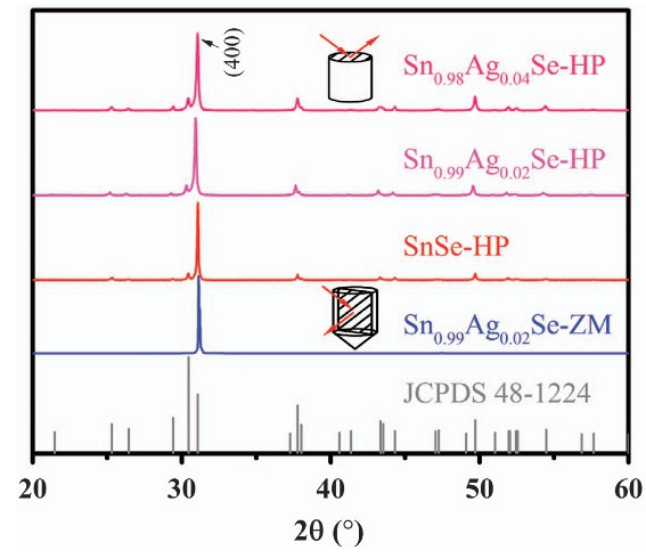

Figure 1 XRD patterns of bulk SnSe and Ag-doped SnSe.

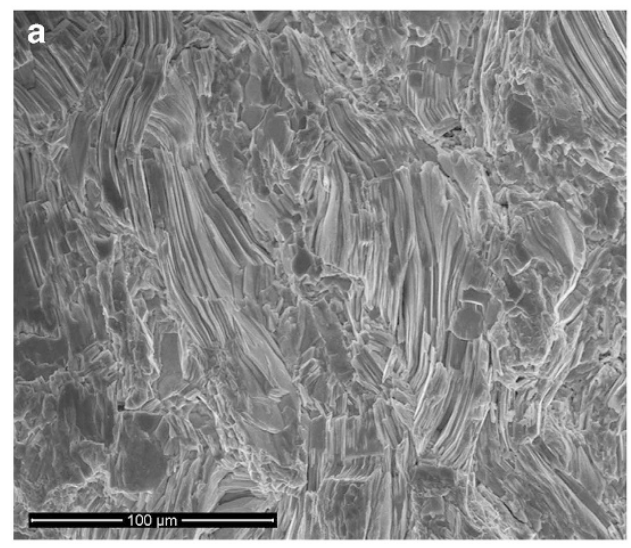

Figure 2 SEM images of bulk $\mathrm{Sn}_{0.99} \mathrm{Ag}_{0.02} \mathrm{Se}$ prepared via HP (a) and ZM (b). ductivity were simultaneously measured using a ZEM-3 (Ulvac Riko, Inc., Kanagawa, Japan) instrument in helium atmosphere from room temperature to $850 \mathrm{~K}$. For thermal diffusivity measurements, the samples were cut and polished into a disk shape of $\Phi 6 \mathrm{~mm} \times 1.5 \mathrm{~mm}$. The thermal conductivity was determined via the relationship $\kappa=D C_{\mathrm{p}} \rho$, where $D, C_{\mathrm{p}}$ and $\rho$ are the thermal diffusivity, heat capacity and density, respectively. The thermal diffusivity was measured using a laser-flash system (LFA457; Netzsch, Selb, Germany) in the temperature range of 300-850 K. The density was measured using the Archimedes method. The heat capacity was obtained from previous reports. ${ }^{11}$ The Hall coefficient $R_{\mathrm{H}}$ was measured at room temperature using a physical properties measurement system (PPMS-9; Quantum Design, San Diego, CA, USA) with magnetic fields in the range of -5 and $5 \mathrm{~T}$. The carrier concentration $n_{\mathrm{H}}$ was calculated via $n_{\mathrm{H}}=1 / e R_{\mathrm{H}}$, where $e$ is the electronic charge. The carrier mobility $\mu$ was calculated via $\mu=\sigma R_{\mathrm{H}}$.

\section{RESULTS AND DISCUSSION}

It has been reported that Ag doping increases the carrier concentration of SnSe, which leads to the enhanced thermoelectric properties. However, the reported optimal doping levels are not consistent with each other. For SCs, the thermoelectric performance increases with an increase in the amount of $\mathrm{Ag}$, and $\mathrm{Sn}_{0.97} \mathrm{Ag}_{0.03}$ Se shows the highest $Z \mathrm{~T}$ of 1.2 at $800 \mathrm{~K} .{ }^{13}$ However, for polycrystalline samples that are prepared via both $\mathrm{HP}$ and SPS, $\mathrm{Sn}_{0.99} \mathrm{Ag}_{0.01}$ Se has better performance than other compositions. ${ }^{21,22}$ Thus, the first step of our experiment is to determine the optimal concentration of Ag. The samples were synthesized via HP.

The XRD patterns of the HP SnSe bulk sample in the planes perpendicular to the pressure direction are shown in Figure 1. All peaks show an excellent match to the standard pattern of SnSe (JCPDS \#48-1224) and can be indexed based on the orthorhombic phase (space group Pnma). Structural anisotropy is clearly observed in the XRD patterns measured along different directions (Supplementary Figure S1) because of its layered crystal structure. No impurity phase was observed within the XRD detection limit.

The SEM image of HP $\mathrm{Sn}_{0.99} \mathrm{Ag}_{0.02} \mathrm{Se}$ is shown in Figure 2a. The layered crystal structure can be observed. Roughly, all crystallites orient in a similar direction. A number of micrometer-sized $\mathrm{Ag}_{2} \mathrm{Se}$ inclusions are observed in $\mathrm{HP} \mathrm{Sn}_{0.99} \mathrm{Ag}_{0.02} \mathrm{Se}$ after polishing the surface (Supplementary Figure S2). The quantitative analysis via energy dispersive spectrometer and electron probe micro-analysis shows that the actual Ag amount in the matrix is one order lower than the nominal composition. This result may explain why the reported carrier concentration of doped samples is considerably smaller than expected. ${ }^{21,22}$

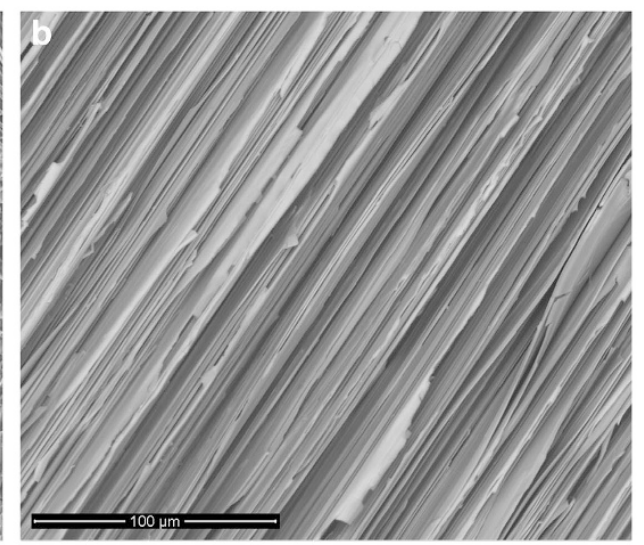


a

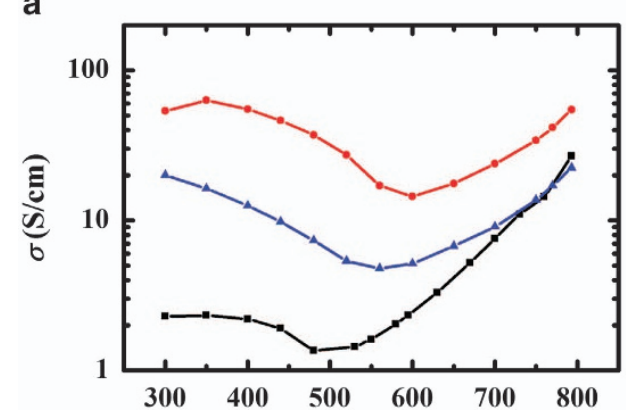

c

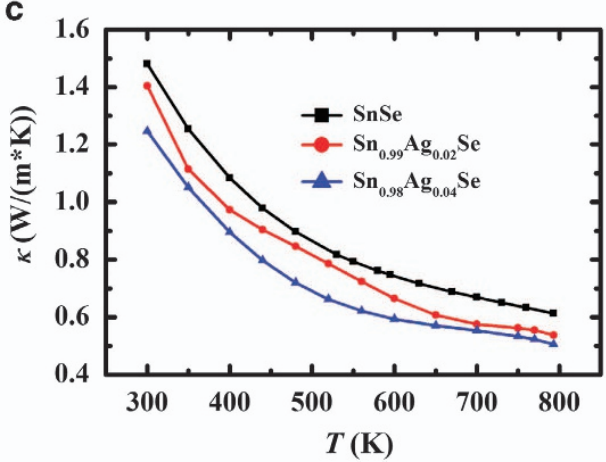

b

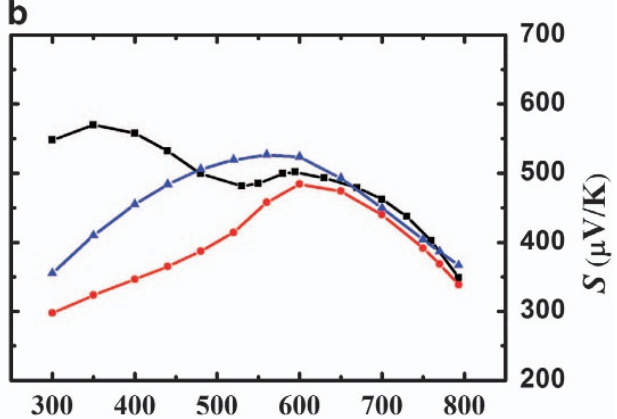

d

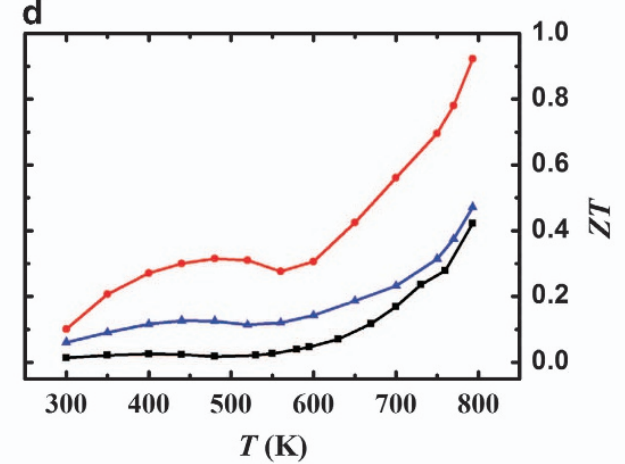

Figure 3 Temperature dependence of the (a) electrical conductivity, (b) Seebeck coefficient, (c) thermal conductivity and (d) $Z T$ values for the $\mathrm{Sn}_{1-\mathrm{x}} \mathrm{Ag} \mathrm{g}_{2 \mathrm{x}} \mathrm{Se}$ samples prepared via HP. The properties were measured perpendicular to the pressing direction.

a

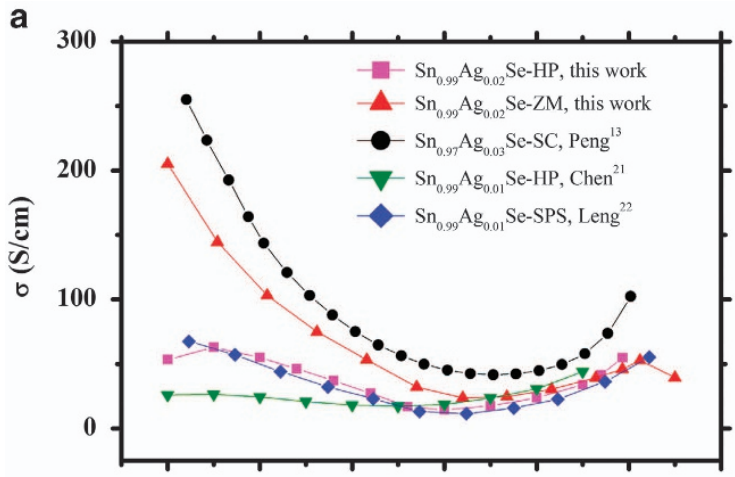

C

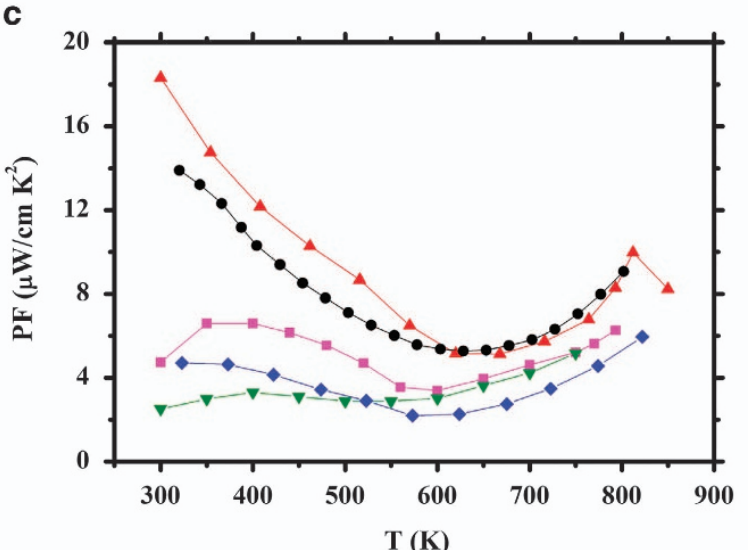

b

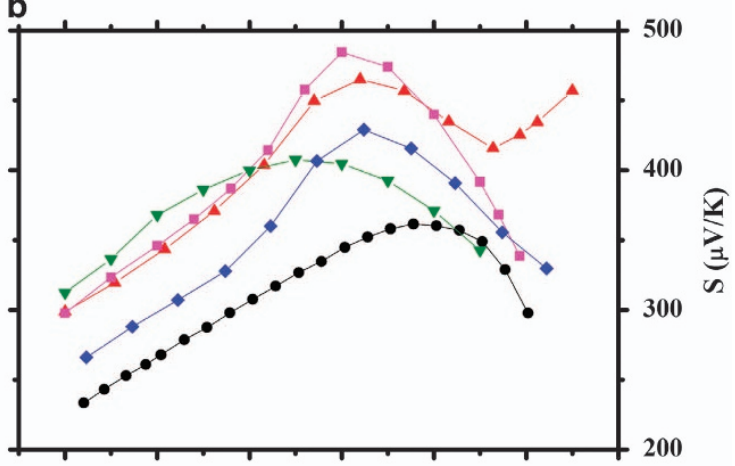

d

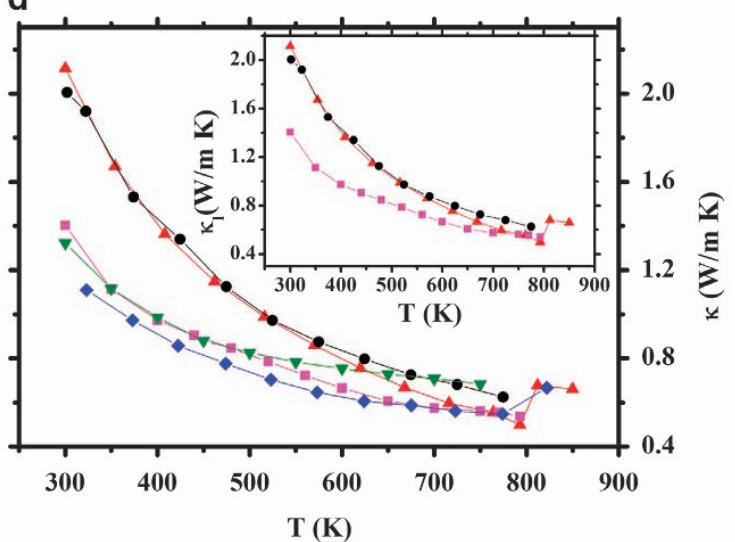

Figure 4 Temperature dependence of the thermoelectric properties for the Ag-doped SnSe samples prepared via different methods. (a) Electrical conductivity, (b) Seebeck coefficients, (c) power factors and (d) thermal conductivity. The lattice thermal conductivity is shown in the inset of $\mathbf{d}$. 
a

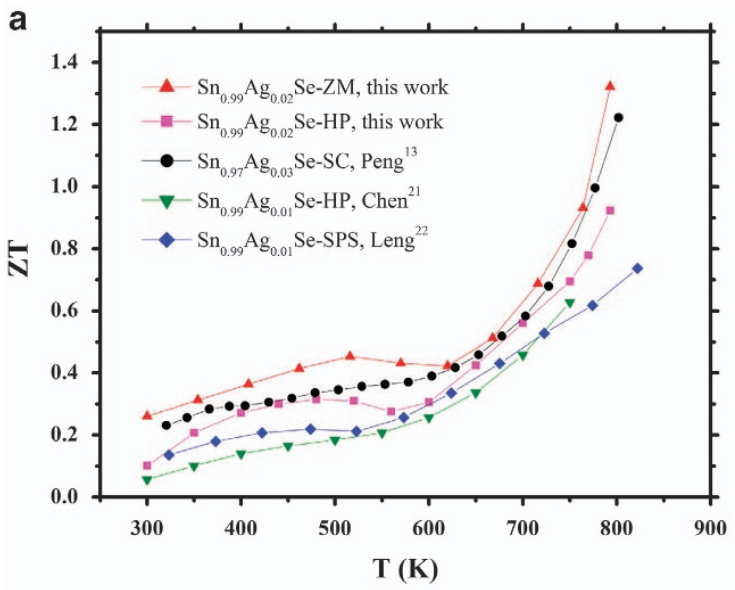

b

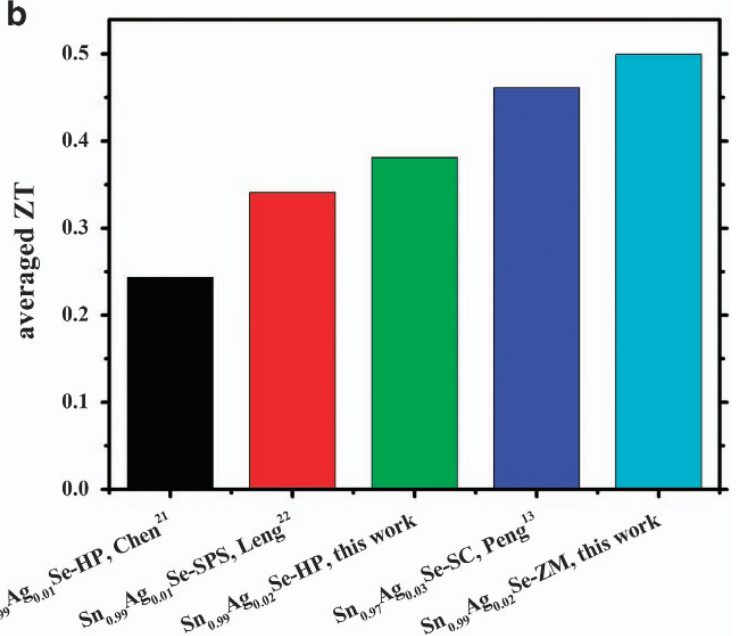

Figure 5 Figure of merit $Z T$ (a) and averaged $Z T$ (b) for the Ag-doped SnSe samples prepared via different methods.

The thermoelectric properties of HP samples were measured perpendicular to the pressure direction because this direction shows a preferred orientation of (400) ( $b c$ plane) based on XRD. The results are shown in Figure 3. The electrical conductivity for undoped SnSe shows an almost the same trend as for the reported SC. The conductivity first decreases with increasing temperature, reaches a minimum at around $480 \mathrm{~K}$, and later increases with further increasing temperature. However, the absolute value is close to that along the $a$ axis for the SC. Ag doping enhances the electrical conductivity, but $\mathrm{Sn}_{0.99} \mathrm{Ag}_{0.02} \mathrm{Se}$ shows higher electrical conductivity than $\mathrm{Sn}_{0.98} \mathrm{Sn}_{0.04} \mathrm{Se}$. The Seebeck coefficient of undoped SnSe decreases with increasing temperature. An anomaly is observed at $\sim 530 \mathrm{~K}$, which may be attributed to the melting of Sn impurity. The Seebeck coefficient is suppressed after Ag doping, which indicates the increase of carrier concentration. However, the Seebeck coefficient for $\mathrm{Sn}_{0.98} \mathrm{Sn}_{0.04} \mathrm{Se}$ is also lower than that of $\mathrm{Sn}_{0.99} \mathrm{Sn}_{0.02} \mathrm{Se}$, which is consistent with the change in electrical conductivity. These results differ from previous reports, which showed that $\mathrm{Ag}$ doping continuously increases the carrier concentration. This phenomenon may be explained by the existence of $\mathrm{Ag}_{2} \mathrm{Se}$ inclusions because $\mathrm{Ag}$ in $\mathrm{Sn}_{0.98} \mathrm{Ag}_{0.04} \mathrm{Se}$ may only form $\mathrm{Ag}_{2} \mathrm{Se}$ inclusions and is not substituted in the matrix. The Seebeck coefficient of doped samples increases with increasing temperature and shows a maximum at around $600 \mathrm{~K}$. Above this temperature, the Seebeck coefficients of undoped and doped samples are close to each other.

The thermal conductivity of all samples decreases with increasing temperature, and it also decreases with increasing $\mathrm{Ag}$, which indicates the enhanced phonon scattering by point defects or the $\mathrm{Ag}_{2} \mathrm{Se}$ inclusions. At room temperature, the thermal conductivity of SnSe is $\sim 1.5 \mathrm{~W} \mathrm{~m}^{-1} \mathrm{~K}$, which is consistent with previously reported values in HP and SPS samples. ${ }^{21,22}$ The lowest thermal conductivity is observed to be $\sim 0.5 \mathrm{~W} \mathrm{~m}^{-1} \mathrm{~K}$ at $793 \mathrm{~K}$ in $\mathrm{Sn}_{0.98} \mathrm{Ag}_{0.04} \mathrm{Se}$. The $Z T$ values are calculated from the measured properties and are plotted in Figure 3d. Due to better electrical properties, $\mathrm{Sn}_{0.99} \mathrm{Ag}_{0.02} \mathrm{Se}$ shows higher $Z T$ values than $\mathrm{Sn}_{0.98} \mathrm{Ag}_{0.04} \mathrm{Se}$ and undoped SnSe. The highest $Z T$ in $\mathrm{Sn}_{0.99} \mathrm{Ag}_{0.02} \mathrm{Se}$ is found to be over 0.9 at $793 \mathrm{~K}$, which is higher than the reported values in polycrystalline SnSe materials. ${ }^{21-29}$ However, this value is still smaller than that of the Ag-doped SnSe SC (1.2).

To explore the method for achieving a high-performance polycrystalline SnSe, we fix the amount of Ag and investigate the impact of synthesis methods on the properties of Ag-doped SnSe samples. The XRD result of the ZM sample is shown in Figure 1. Only the peak for the (400) plane is clearly observed, which suggests a considerably better degree of texturing. The SEM image of the ZM sample (in Figure 2b) also shows a higher texturing degree than the HP sample.

In Figure 4, we compare the thermoelectric properties of $\mathrm{Sn}_{0.99} \mathrm{Ag}_{0.02} \mathrm{Se}$ prepared via $\mathrm{ZM}$ and HP with the best results of the SC and polycrystalline samples produced via HP and SPS. ${ }^{13,21,22}$ In Figure 4a, the room temperature electrical conductivity for Ag-doped SnSe varies in a very large range, from $\sim 40 \mathrm{~S} / \mathrm{cm}$ for the HP sample to $\sim 300 \mathrm{~S} / \mathrm{cm}$ for the SC. In addition, the samples fall into two categories: the SC and ZM samples show high conductivity that is larger than $200 \mathrm{~S} \mathrm{~cm}^{-1}$, while the HP and SPS samples show conductivity that is less than $100 \mathrm{~S} \mathrm{~cm}^{-1}$. The temperature dependence of Seebeck coefficients for all samples is similar to Figure 4b, as mentioned above. The difference in Seebeck coefficient values for all samples is much smaller than that in electrical conductivity. At room temperature, the Seebeck coefficient changes from $\sim 240$ to $\sim 320 \mu \mathrm{V} \mathrm{K}^{-1}$, and the SC shows smaller Seebeck coefficients than other polycrystalline samples. The power factors are calculated and shown in Figure 4c. Due to the high conductivity, the SC and ZM samples show larger power factors than other samples. These two samples show a very similar temperature dependence, and the power factors for the ZM sample are larger than those for the SC at low temperature due to high Seebeck coefficients.

The thermal conductivity is shown in Figure 4c. Polycrystalline samples show a lower thermal conductivity than the Ag-doped SC. Furthermore, the thermal conductivity of the samples falls into two categories: the SC and ZM samples with a high thermal conductivity at room temperature, and the HP and SPS samples with a lower thermal conductivity. The thermal conductivity of the ZM sample is close to that of the SC below $550 \mathrm{~K}$. Above this temperature, the ZM sample shows lower thermal conductivity. At $793 \mathrm{~K}$, the lowest thermal conductivity is $0.50 \mathrm{~W} \mathrm{~m}^{-1} \mathrm{~K}$ for the $\mathrm{ZM}$ sample compared with $0.62 \mathrm{~W} \mathrm{~m}^{-1} \mathrm{~K}$ for the SC. The lattice thermal conductivity is estimated by subtracting the electronic contribution from the total thermal conductivity $\kappa_{1}=\kappa-\kappa_{\mathrm{e}}$, where the electronic contribution $\kappa_{\mathrm{e}}$ is obtained via the Wiedemann-Franz law, $\kappa_{\mathrm{e}}=L \sigma T$. The Lorenz number $L$ is calculated using the accepted approach of fitting the Seebeck data to the reduced chemical potential. ${ }^{31,32}$ In this calculation, $L$ is in the range of $1.45-1.55 \times 10^{-8} \mathrm{~V}^{2} \mathrm{~K}^{-2}$ (Supplementary Figure S3). As 
shown in the inset of Figure $4 \mathrm{~d}, \kappa_{1}$ for the HP sample is considerably lower than that for the SC. It is difficult to differentiate $\kappa_{\mathrm{l}}$ for the ZM sample and the SC below $600 \mathrm{~K}$, and the ZM sample shows a minimum $\kappa_{1}$ of $0.44 \mathrm{~W} \mathrm{~m}^{-1} \mathrm{~K}$, which is also lower than 0.54 for the SC.

Due to better electrical properties at low temperature and lower thermal conductivity at high temperature, the $\mathrm{ZM}$ sample shows higher $Z T$ values than the $\mathrm{SC}$ in the entire temperature range, as shown in Figure 5a. The highest ZT for the ZM sample is 1.3 at $793 \mathrm{~K}$, compared with 1.2 for the SC. This result shows that the polycrystalline SnSe material has better performance than the SC with a similar composition. Conversely, the HP and SPS samples show smaller ZT values than the SC, even though they show lower thermal conductivity. Because the thermoelectric conversion efficiency is defined by the average $Z T$ over the entire working temperature range, we calculated the average $Z T$ based on the relationship $Z T_{\text {ave }}=\frac{1}{T_{h}-T_{c}} \int_{T_{c}}^{T_{h}} Z T \mathrm{~d} T$, where $T_{h}$ and $T_{c}$ are the hot-side and cold-side temperatures, respectively. The average $Z T$ values of Ag-doped SnSe materials are shown in Figure 5b. In the temperature range of $300-800 \mathrm{~K}$, the average $Z T$ of our $\mathrm{ZM} \mathrm{Sn}_{0.99} \mathrm{Ag}_{0.02} \mathrm{Se}$ sample is $\sim 0.5$ and higher than that of all other samples.

The compressed samples show lower thermal conductivity (Figure 4d). The reason for the poor thermoelectric performance of the compressed samples is poor electrical conductivity (Figure 4c). In Figure 6, we plot the carrier mobility with carrier concentration for the Ag-doped samples. It can be seen that the polycrystalline samples are divided into two categories, similar to what we observed for electrical and thermal conductivities. The three squares represent mobility along

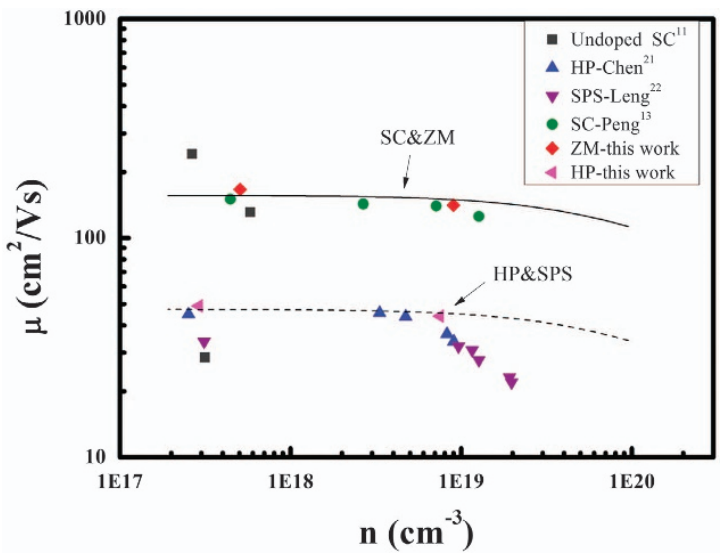

Figure 6 Room temperature Hall mobility for the Ag-doped SnSe samples prepared via different methods. The two lines were calculated using SPB by adjusting $\mu_{0}$. the $a, b$ and $c$ axis of the SC. The mobility of ZM samples is close to that along the $b$ axis of the Ag-doped SnSe SCs, and approaches the best one for undoped SC along the $b$ axis. Even though these properties are measured along the direction where (400) plane dominates the XRD pattern, the compressed samples only show low mobility, which is similar to the value along the $a$ axis of the SC. To explore the difference, we calculated the orientation factor $F$ of the (400) plane for all samples. $F$ is defined as

$$
\begin{aligned}
& f=\frac{p-p_{0}}{1-p_{0}} \\
& p=\frac{\sum I(h 00)}{\sum I(h k l)} \\
& p_{0}=\frac{\sum I_{0}(h 00)}{\sum I_{0}(h k l)}
\end{aligned}
$$

where $I$ and $I_{0}$ are the intensities of XRD reflection peaks for oriented and non-oriented samples, respectively; $p$ and $p_{0}$ are the ratios of the sum of intensities of the $(h 00)$ reflection to that of all $(h k l)$ reflections for oriented and non-oriented samples, respectively. ${ }^{33}$ The standard diffraction pattern of SnSe is used to calculate $p_{0}$. For non-oriented polycrystalline samples, $F$ is 0 , while $F$ is 1 along the (h00) plane in the SC. The calculated orientation factors along the (400) plane are listed in Table 1, and a clear difference in orientation factors can be observed. For the compressed samples, the $F(400)$ is $\sim 0.5$ or even lower. For this degree of texturing, misoriented crystallites may dominate, and the carrier along the $a$ axis may still significantly contribute to transport. The results of the ZM samples suggest that the high orientation is essential for obtaining high mobility in polycrystalline SnSe. Because the total thermal conductivity of polycrystalline SnSe is lower than that of the Ag-doped SC (Figure 4d), this point becomes a key factor for achieving high thermoelectric performance of polycrystalline SnSe.

\section{CONCLUSIONS}

The thermoelectric properties of Ag-doped SnSe polycrystals were investigated. Large differences in electrical and thermal conductivities were observed between the samples produced via ZM and HP. The orientation factors are calculated and suggest that the texturing degree is the origin. The polycrystalline $\mathrm{Sn}_{0.99} \mathrm{Ag}_{0.02} \mathrm{Se}$ prepared via $\mathrm{ZM}$ shows better thermoelectric performance than the Ag-doped SC in the entire temperature range. A peak $Z T$ of 1.3 at $793 \mathrm{~K}$ and an average $Z T$ of $\sim 0.5$ are observed in $\mathrm{ZM} \mathrm{Sn}_{0.99} \mathrm{Ag}_{0.02} \mathrm{Se}$, both of which are better than those in the Ag-doped SC. Our results suggest that a high texturing degree is essential for obtaining high carrier mobility and therefore high thermoelectric performance of polycrystalline SnSe.

Table 1 Room temperature carrier concentration $(n)$, carrier mobility $(\mu)$, electrical conductivity $(\sigma)$, Seebeck coefficient $(S)$ and the orientation factor $\mathrm{F}$ for the (400) plane in the diffraction angle range from $20^{\circ}$ to $60^{\circ}$ of the SnSe and Ag-doped SnSe samples

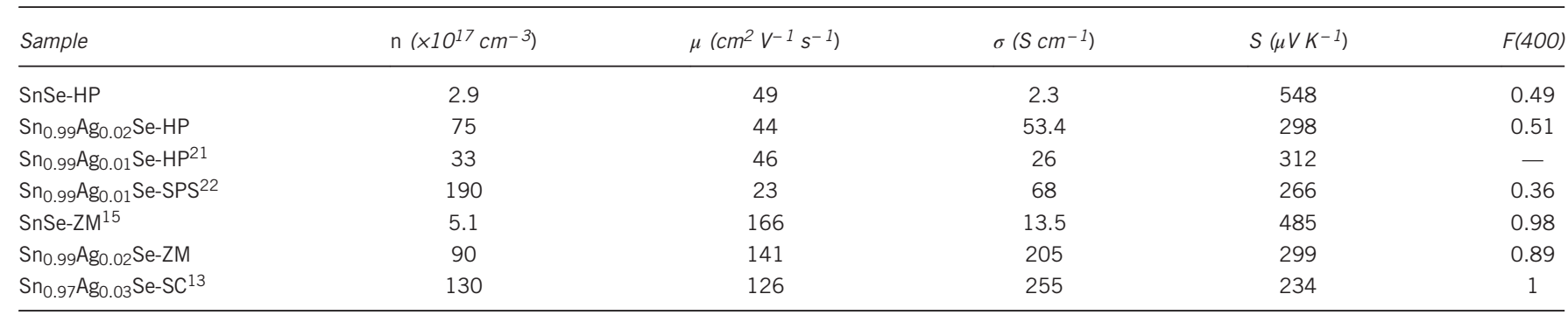




\section{CONFLICT OF INTEREST}

The authors declare no conflict of interest.

\section{ACKNOWLEDGEMENTS}

This work was supported by the National Nature Science Foundation of China (NSFC No 11304327, 11404348, 11404350 and 11234012), the Ningbo Municipal Natural Science Foundation (2015A610031), the Ningbo Science and Technology Innovation Team (Grant No 2014B82004) and the Zhejiang Provincial Science Fund for Distinguished Young Scholars (LR16E020001).

\section{PUBLISHER'S NOTE}

Springer Nature remains neutral with regard to jurisdictional claims in published maps and institutional affiliations.

1 Bell, L. E. Cooling, heating, generating power, and recovering waste heat with thermoelectric systems. Science 321, 1457 (2008).

2 Snyder, G. J. \& Toberer, E. S. Complex thermoelectric materials. Nat. Mater. 7, 105 (2008).

3 Rowe, D. M. Handbook of Thermoelectric (CRC Press: Boca Raton, FL, USA, 1995).

4 Kanatzidis, M. G. Nanostructured thermoelectrics: the new paradigm? Chem. Mater 22, 648 (2010).

5 Dresselhaus, M. S., Chen, G., Tang, M. Y., Yang, R. G., Lee, H., Wang, D. Z., Ren, Z. F., Fleurial, J.-P. \& Gogna, P. New directions for low-dimensional thermoelectric materials. Adv. Mater. 19, 1043 (2007).

6 Heremans, J. P., Jovovic, V., Toberer, E. S., Saramat, A., Kurosaki, K., Charoenphakdee, A., Yamanaka, S. \& Snyder, G. J. Enhancement of thermoelectric efficiency in PbTe by distortion of the electronic density of states. Science 321, 554 (2008).

7 Pei, Y. Z., Shi, X., LaLonde, A., Wang, H., Chen, L. \& Snyder, G. J. Convergence of electronic bands for high performance bulk thermoelectrics. Nature 473, 66 (2011).

8 Biswas, K., He, J., Blum, I. D., WU, C.-I., Hogan, T. P., Seidman, D. N., Dravid, V. P. \& Kanatizids, M. G. High-performance bulk thermoelectrics with all-scale hierarchical architectures. Nature 489, 414 (2012).

9 Tan, G. J., Shi, F. Y., Hao, S. Q., Zhao, L.-D., Chi, H., Zhang, X. M., Uher, C., Wolverton, C., Dravid, V. P. \& Kanatizidis, M. G. Non-equilibrium processing leads to record high thermoelectric figure of merit in PbTe-SrTe. Nat. Commun. 7, 12167 (2016).

10 Kim, S. I., Lee, K. H., Mun, H. A., Kim, H. S., Hwang, S. W., Roh, J. W., Yang, D. J., Shin, W. H., Li, X. S., Lee, Y. H., Snyder, G. J. \& Kim, S. W. Dense dislocation arrays embedded in grain boundaries for high-performance bulk thermoelectrics. Science $\mathbf{3 4 8}$, 109 (2015).

11 Zhao, L.-D., Lo, S.-H., Zhang, Y., Sun, H., Tan, G. J., Uher, C., Wolverton, C., Dravid, V. P. \& Kanatzidis, M. G. Ultralow thermal conductivity and high thermoelectric figure of merit in SnSe crystals. Nature 508, 373 (2014).

12 Zhao, L.-D., Tan, G. J., Hao, S., He, J., Pei, Y., Chi, H., Wang, H., Gong, S., Xu, H., Dravid, V. P., Uher, C., Snyder, G. J., Wolverton, C. \& Kanatzidis, M. G. Ultrahigh power factor and thermoelectric performance in hole-doped single-crystal SnSe. Science 351, 141 (2016).

13 Peng, K. L., Lu, X., Zhan, H., Hui, S., Tang, X., Wang, G., Dai, J., Uher, C., Wang, G. \& Zhou, X. Broad temperature plateau for high ZTs in heavily doped p-type SnSe single crystals. Energy Enviorn. Sci. 9, 454 (2016).

14 Wei, P.-C., Bahttacharya, S., He, J., Neeleshwar, S., Podila, R., Chen, Y. Y. \& Rao, A. M. The intrinsic thermal conductivity of SnSe. Nature 539, E1 (2016).

15 Fu, Y. J., Xu, J. T., Liu, G.-Q., Yang, J. K., Tan, X. J., Liu, Z., Qin, H. M., Shao, H. Z., Jiang, H. C., Liang, B. \& Jiang, J. Enhanced thermoelectric performance in p-type polycrystalline SnSe benefiting from texture modulation. J. Mater. Chem. C 4, 1201 (2016).
16 Popuri, S. R., Pollet, M., Decourt, R., Morrison, F. D., Bennett, N. S. \& Bos, J. W. G. Large thermoelectric power factors and impact of texturing on the thermal conductivity in polycrystalline SnSe. J. Mater. Chem. C 4, 1685 (2016).

17 Sassi, S., Candolfi, C., Vaney, J.-B., Ohorodniichuk, V., Masschelein, P., Dauscher, A. \& Lenoir, B. Assessment of the thermoelectric performance of polycrystalline p-type SnSe. Appl. Phys. Lett. 104, 212105 (2014).

18 Serrano-Sánchez, F., Gharsallah, M., Nemes, N. M., Mompean, F. J., Martínez, J. L. \& Alonso, J. A. Record Seebeck coefficient and extremely low thermal conductivity in nanostructured SnSe. Appl. Phys. Lett. 106, 083902 (2015).

19 Li, Y. L., Shi, X., Ren, D. D., Chen, J. K. \& Chen, L. D. Investigation of the anisotropic thermoelectric properties of oriented polycrystalline SnSe. Energies 8, 6275 (2015).

20 Li, Y. W., Li, F., Dong, J. F., Ge, Z. H., Kang, F. Y., He, J. Q., Du, H. D., Li, B. \& Li, J.-F. Enhanced mid-temperature thermoelectric performance of textured SnSe polycrystals made of solvothermally synthesized powders. J. Mater. Chem. C 4, 2047 (2016).

21 Chen, C.-L., Wang, H., Chen, Y. Y., Day, T. \& Snyder, G. J. Thermoelectric properties of p-type polycrystalline SnSe doped with Ag. J. Mater. Chem. A 2, 11171 (2014).

22 Leng, H. Q., Zhou, M., Zhao, J., Han, Y. M. \& Li, L. F. Optimization of thermoelectric performance of anisotropic Agx $\mathrm{Sn}_{1-\mathrm{x}} \mathrm{Se}$ compounds. J. Electronic Mater. 45, 527 (2016).

23 Chere, E. K., Zhang, Q., Dahal, K., Cao, F., Mao, J. \& Ren, Z. Studies on thermoelectric figure of merit of Na-doped p-type polycrystalline SnSe. J. Mater. Chem. A 4, 1848 (2016)

24 Wei, T.-R., Wu, C.-F., Zhang, X., Tan, Q., Sun, L., Pan, Y. \& Li, J.-F. Thermoelectric transport properties of pristine and $\mathrm{Na}$-doped $\mathrm{SnSe}_{1-x} \mathrm{Te}_{x}$ polycrystals. Phys. Chem. Chem. Phys. 17, 30102 (2015).

25 Leng, H.-Q., Zhou, M., Zhao, J., Han, Y.-M. \& Li, L.-F. The thermoelectric performance of anisotropic SnSe doped with Na. RSC Adv. 6, 9112 (2016).

26 Wei, T.-R., Tan, G. J., Zhang, X. M., Wu, C.-F., Li, J.-F., Dravid, V. P., Snyder, G. J. \& Kanatzidis, M. G. Distinct impact of alkali-ion doping on electrical transport properties of thermoelectric p-type polycrystalline SnSe. J. Am. Chem. Soc. 138, 8875 (2016).

27 Zhang, Q., Chere, E. K., Sun, J., Cao, F., Dahal, K., Chen, S., Chen, G. \& Ren, Z. F. Studies on thermoelectric properties of n-type polycrystalline SnSe1-xSx by iodine doping. Adv. Energy Mater. 5, 1500360 (2015).

28 Wang, X., Xu, J. T., Liu, G. Q., Fu, Y. J., Liu, Z., Tan, X. J., Shao, H. Z., Jiang, H. C., Tan, T. Y. \& Jiang, J. Optimization of thermoelectric properties in n-type SnSe doped with BiCl3. Appl. Phys. Lett. 108, 083902 (2016).

29 Han, Y.-M., Zhao, J., Zhou, M., Jiang, X.-X., Leng, H. \& Li, L.-F. Thermoelectric performance of SnS and SnS-SnSe solid solution. J. Mater. Chem. A 3, 4555 (2015).

$30 \mathrm{Li}$, J. C., Li, D., Qin, X. Y. \& Zhang, J. Enhanced thermoelectric performance of p-type SnSe doped with Zn. Scr. Mater. 126, 6 (2017).

31 Johnsen, S., He, J., Androulakis, J., Dravid, V. P., Todorov, I., Chung, D. Y. \& Kanatzidis, M. G. Nanostructures boost the thermoelectric performance of PbS. J. Am. Chem. Soc. 133, 3460 (2011).

32 He, J., Xu, J. T., Tan, X. J., Liu, G.-Q., Shao, H. Z., Liu, Z., Jiang, H. C. \& Jiang, J. SnTe/ AgSbSe2 nanocomposite as a promising lead-free thermoelectric material. J. Materiomics 2, 165 (2016).

33 Lotgering, F. K. Topotactical reactions with ferrimagnetic oxides having hexagonal crystal structures-I. J. Inorg. Nucl. Chem. 9, 113 (1959).

This work is licensed under a Creative Commons Attribution 4.0 International License. The images or other third party material in this article are included in the article's Creative Commons license, unless indicated otherwise in the credit line; if the material is not included under the Creative Commons license, users will need to obtain permission from the license holder to reproduce the material. To view a copy of this license, visit http:// creativecommons.org/licenses/by/4.0/

(C) The Author(s) 2017

Supplementary Information accompanies the paper on the NPG Asia Materials website (http://www.nature.com/am) 